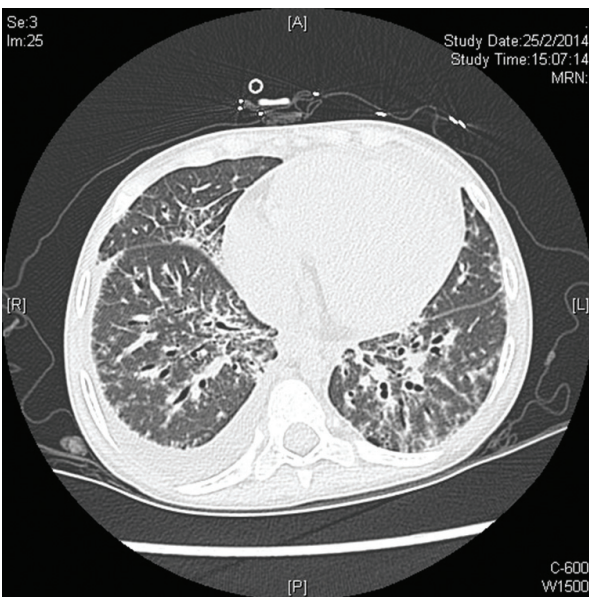

Figure 2

Disclosure of Interests: None declared

DOI: 10.1136/annrheumdis-2019-eular.5376

\section{AB0985 EVALUATION OF SERUM CALPROTECTIN (MRP-8/MRP- 14) LEVEL IN PATIENTS WITH JUVENILE IDIOPATHIC ARTHRITIS}

Tetiana Marushko ${ }^{1}$, Yuliia Holubovska ${ }^{1}$, Yurii Marushko $2 .{ }^{1}$ Shupyk National Medical Academy of Postgraduate Education, Pediatrics №2, Kyiv, Ukraine; ${ }^{2}$ Bogomolets National Medical University, Pediatrics postgraduate education, Kyiv, Ukraine

Background: Evaluation of inflammatory activity is an important element in the management of patients with juvenile idiopathic arthritis (JIA), for which C-reactive protein (CRP) and erythrocyte sedimentation rate (ESR) are traditionally used. However, they might be uninformative in case of subclinical inflammation. The serum level of calprotectin MRP-8/MRP-14 (sCal) correlates well with arthritis activity, as it is produced by activated cells directly in synovia.

Objectives: We evaluate the level of sCal in patients with JIA depending on the type of therapy in order to assess comprehensively the disease activity for further treatment correction.

Methods: 74 patients with JIA were examined, 18 of them had oligoarticular disease subtype, 39 - polyarticular, 17 - systemic. The mean age was $11.3 \pm 0.4$ years; the disease duration was $5.2 \pm 0.4$ years. Among them, there were $49(66 \%)$ females and $25(34 \%)$ males. All patients were divided into 2 groups depending on the therapy type. Group I consisted of 33 children treated with methotrexate, while 11 of them were in a state of clinical remission. Group II included 41 children treated with biologic DMARDs (adalimumab, etanercept, tocilizumab), while 14 of them achieved clinical remission. All children had normal levels of CRP and ESR. Quantitative indicators distribution is given as a median [5th; 95th percentile], the calculations were carried out using the Mann-Whitney $U$ test.

Results: Level of sCal in the active disease stage in children of Group I was $8,750 \mathrm{ng} / \mathrm{ml}[3,700 ; 17,100]$, while sCal level in Group II was 2,900 $\mathrm{ng} / \mathrm{ml}[1,200 ; 24,900]$; sCal level in children of Group I which achieved clinical remission $-3,400 \mathrm{ng} / \mathrm{ml}[1,200 ; 6,000]$, and the same indicator in Group II $-1,000 \mathrm{ng} / \mathrm{ml}[100 ; 2,800]$. sCal level was significantly higher in the group of patients who did not receive biologic DMARDs, both in the active stage of disease $(p=0.000006, U=71.5)$ and in the stage of clinical remission $(p=0.00034, U=11)$. sCal level is $5,800 \mathrm{ng} / \mathrm{ml}$ less in patients with active stage of disease and $2,400 \mathrm{ng} / \mathrm{ml}$ less in patients with clinical remission, both treated with biologic DMARDs. In addition, the level of sCal is 5.5 times higher in our patients $(3,300 \mathrm{ng} /$ $\mathrm{ml})$ compared with healthy children $(600 \mathrm{ng} / \mathrm{ml})(\mathrm{p}=0.015)$. The moderate positive correlation of sCal and JADAS-27 activity index ( $r$-Spearman's $=0.58$ ) was credibly established.

Conclusion: The level of $\mathrm{sCal}$ can reflects the degree of inflammatory activity in JIA, it is significantly higher in the group of patients who did not receive biologic DMARDs in the treatment regimen, both in the active disease stage $(p=0.000006, U=71.5)$ and in the stage of clinical remission $(p=0.00034, U=11)$, which indicates the effectiveness of biologic DMARDs in the treatment of JIA. We assume that it would be appropriate to estimate the serum calprotectin level in the comprehensive analysis of clinical status in JIA patients for the further correction of therapy.

Disclosure of Interests: None declared

DOI: 10.1136/annrheumdis-2019-eular.4748

\section{\begin{tabular}{|l|l} 
AB0986 & VITAMIN D LEVEL AND BONE MINERAL DENSITY IN
\end{tabular} PATIENTS WITH JUVENILE RHEUMATOID ARTHRITIS}

Tetiana Marushko ${ }^{1}$, Yuliia Holubovska ${ }^{1}$, Yurii Marushko2. ${ }^{1}$ Shupyk National Medical Academy of Postgraduate Education, Pediatrics №2, Kyiv, Ukraine; ${ }^{2}$ Bogomolets National Medical University, Pediatrics postgraduate education, Kyiv, Ukraine

Background: An impaired bone metabolism is observed in patients with juvenile idiopathic arthritis (JIA), associated both with the activation of pro-inflammatory cytokines and use of steroid medications. The majority of children with JIA have low vitamin D level, which may complicate the disease course. We regard the monitoring of bone mineral density (BMD) in patients with juvenile rheumatoid arthritis (JRA) should be complemented with an additional assessment of calcidiol $(25(\mathrm{OH}) \mathrm{D})$ serum level as an indicator of BMD and a criterion of successful therapy.

Objectives: To evaluate the bone densitometry data and calcidiol level in patients with JRA in order to estimate the osteopenic syndrome and the advisability of prescribing the vitamin $\mathrm{D}$ additional doses.

Methods: The calcidiol level and BMD data in 65 patients with JIA were assessed (41 girls and 24 boys). All children were divided into 2 groups, depending on the therapy type. Group I consisted of patients who received methotrexate $(n=37)$, Group $\|$ - patients who received the biological DMARDs $(n=28)$, namely tocilizumab ( 6 patients), adalimumab (20 patients) and etanercept (2 patients). At the time of the study, 14 children of the Group II were prescribed the biological DMARDs only, while the 12 patients were in the state of clinical remission unlike the 7 patients of Group I. It should be mentioned that the majority of children in the Group II had high disease activity degrees before the start of treatment with biological DMARDs. In order to show the correlation between BMD and disease activity, CJADAS-27 score result for the last 6 months was estimated. The data were processed using Pearson's chisquared test and Spearman's Rank correlation coefficient.

Results: The study revealed, 60 children (92\% of all patients) showed vitamin $D$ insufficiency, that mainly manifested by decreased calcidiol level (from 21 to $29 \mathrm{ng} / \mathrm{ml}$ ), only 8 children $(12 \%)$ showed calcidiol level deficiency $(<20 \mathrm{ng} / \mathrm{ml})$. A significant difference was found in the BMD results depending on the therapy type $(\alpha 2=10.05 ; p<0.01)$ using Pearson's chi-squared test. As a result, children who received biological DMARDs, demonstrated significantly better results according to BMD assessment data. A direct association of moderate strength was found (the correlation coefficient ( $r$-Spearman's) is 0,39). The strong negative association ( $r$ Spearman's was -0,72) was observed between BMD assessment data and CJADAS-27 score result, which confirmed our statement - high disease activity affects the bone tissue mineralization. There was a weak negative association between 25(OH)D level and cJADAS-27 score result (r-Spearman's was -0,15)

Conclusion: There was a paucity of vitamin D level in $92 \%$ of patients with JRA, in $60 \%$ secondary osteopenic syndrome. It was revealed that patients, who receive biological DMARDs in integrated treatment, demonstrate significantly better results according to BMD assessment data. Calcidiol level has a moderate effect on BMD, while BMD strongly depends on disease activity degree according to the Spearman's Rank correlation coefficient.

Disclosure of Interests: None declared

DOI: 10.1136/annrheumdis-2019-eular.6030

\section{AB0987 \\ CHOICE AND SWITCH BETWEEN BIOLOGICAL AGENTS IN NON-SYSTEMIC JUVENILE IDIOPATHIC ARTHRITIS (JIA)}

Anna Ignatova ${ }^{1}$, Nina Seylanova ${ }^{1}$, Elena Zholobova ${ }^{2} .{ }^{1}$ I.M. Sechenov First Moscow State Medical University (Sechenov University), Moscow, Russian Federation; ${ }^{2}$ I.M. Sechenov First Moscow State Medical University (Sechenov University), Paediatric Rheumatology, Moscow, Russian Federation

Background: Over the past few decades, biologic therapy has significantly improved the prognosis in children with JIA. The issue of optimal and personalised biologics prescription and the problem of switching between drugs are relevant questions of modern paediatric rheumatology. 
Objectives: The aim of our retrospective observation was to describe trends in the biologics prescription in first and subsequent lines for the treatment of non-systemic JIA.

Methods: We recruited 252 patients with non-systemic JIA, who received medical treatment with biologics at the Department of Paediatric Rheumatology of Sechenov University from January 2015 to December 2017. $18 \%$ of them $(n=46)$ had chronic anterior uveitis at the moment of biologic therapy initiation. Eye involvement influenced treatment decisions in these patients, and therefore they were excluded from the analysis. 206 children with non-systemic JIA and without uveitis were included in a study group.

Patients' characteristics: 128 girls and 78 boys (1,6/1). Mean age 13,5 \pm 3,8 years, age of disease onset $7,8 \pm 4,2$ years; mean disease duration before biologic therapy initiation $3,3 \pm 3,4$ years. JIA categories: 135 $(65,5 \%)$ had polyarticular RF-, $29(14,1 \%)$ - oligoarticular, $26(12,6 \%)$ enthesitis-related, $9(4,4 \%)$ - polyarticular RF+, and $7(3,4 \%)$ - psoriatic subtype. At baseline, 204 patients (99,0\%) received concomitant therapy with conventional DMARDs: 175 children (85,0\%) received Methotrexate (MTX), $3(1,5 \%)$ - combination of MTX and sulfasalazin (SSZ), $9(4,4 \%)$ - SSZ, $9(4,4 \%)$ - leflunomid, $4(1,9 \%)$ - combination of MTX and leflunomid, $4(1,9 \%)$ - cyclosporine A. Two children $(1,0 \%)$ received biologics as monotherapy.

Results: As a first biologic abatacept was used in 26 patients, adalimumab in 14, tocilizumab in 6 and infliximab in 4 . Etanercept was prescribed to 156 patients $(75,7 \% \pm 3,5)$ with non-systemic JIA, which was significantly more frequently than other biologics together - $50(24,3 \% \pm$ $6,2) \quad(t=7,1 ; P>99,7 \%)$. Later 24 children (11,7\%) were switched from a first to a second biologic agent. The main reason for switching $(n=14)$ was inefficacy of the first-line drug. When etanercept was ineffective, abatacept $(n=3)$ and adalimumab $(n=5)$ were prescribed as second-line biologics. Etanercept was used as a second-line biologic in 6 children, who were initially treated with other biologic agents. The other reason for switching was the appearance of chronic anterior uveitis $(n=7)$ : 6 of these children were initially treated with etanercept, 1 with abatacept; they were switched to adalimumab $(n=6)$ and abatacept $(n=1)$. In 2 cases adverse events were observed - one episode of intrathoracic lymphatic nodes tuberculosis in a patient receiving etanercept, and one allergic reaction (rush, asphyxia) after the infliximab injection. At the end of our observation etanercept was chosen as treatment for $148(71,8 \%)$ patients with non-systemic JIA.

Conclusion: Thus, etanercept was preferred biologic agent in the treatment of non-systemic JIA. The presence of uveitis requires a different treatment approach.

\section{REFERENCES}

[1] Kearsley-Fleet, et al. Factors associated with choice of biologic among children with JIA: results from two UK paediatric biologic registers. Rheumatology (Oxford).2016;55:1556-1565

Disclosure of Interests: Anna Ignatova: None declared, Nina Seylanova: None declared, Elena Zholobova Grant/research support from: Roche, Pfizer, Novartis, Consultant for: Pfizer, AbbVie, Roche, BMS, Novartis, MSD, Speakers bureau: Pfizer, AbbVie, Roche, BMS, Novartis, MSD DOI: 10.1136/annrheumdis-2019-eular.3462

\section{AB0988 THE ASSOCIATION BETWEEN CLINICAL FEATURES AND ANALYSIS OF MEFV GENE IN 20 JAPANESE PATIENTS WITH FAMILIAL MEDITERRANEAN FEVER (FMF)}

Tatsuo Ishizuka ${ }^{1}$, Kei Fujioka ${ }^{1}$, Ichiro Mori ${ }^{1}$, Tmofumi Takeda ${ }^{1}$, Saori Inui ${ }^{1}$, Kouichiro Taguchi ${ }^{2}$, Takahide lkeda ${ }^{2}$, Hiroyuki Morita ${ }^{2}$, Tomoko Toma ${ }^{3}$, Akihiro Yachie ${ }^{3} .{ }^{1}$ Gifu Municipal Hospital, Center of General Internal Medicine and Rheumatology, Gifu, Japan; ${ }^{2}$ Gifu University Graduate School of Medicine, Department of General Internal Medicine, Gifu, Japan; ${ }^{3}$ Kanazawa University Graduate School of Medicine, Department of Pediatrics, Kanazawa, Japan

Background: FMF is recessive systemic auto inflammatory disorder characterized by recurrent fever, peritonitis, pleuritis, pericarditis and arthritis accompanied with skin rash. Mutation of MEFV gene encoding pyrin resulted in inflammasome activation and the uncontrolled production of IL$1 \beta$. Overview of pathogenesis, clinical features and management in Japanese patients with FMF had been reported ${ }^{1}$. However, the differences of clinical features between mutated and non-mutated of MEFV still remain unclear.

Objectives: We have analyzed 20 Japanese patients with FMF to clarify the association between various clinical features and mutation of MEFV.
Methods: Genomic DNA were purified from white blood cells in 20 FMF patients, and mutated MEFV has been explored. We have analyzed MEFV, TNFRSF1A, MVK and NLRP3 genes in 20 patients with FMF Therefore, we excluded another autoinflammatory diseases such as TNF receptor-associated syndrome (TRAPS), mevalonate kinase deficiency and cryopyrin-associated periodic syndrome. Clinical symptoms and laboratory data were analyzed around onset time. Each patient had been treated with colchicine (0.5-2 mg).

Results: Characteristics of Patients with FMF (13 female/7 male) were as follows; Onset time were $0-53$ years old (17.5 \pm 12.2$)$, and teen aged patients were most. Frequencies of clinical symptoms such as periodic fever, headache, arthralgia, abdominal pain, chest pain, myalgia, and cervical lymph nodes swelling were 20/20,7/20,6/20,5/20,4/20,2/20 and 1/ 20 , respectively (double symptoms were observed). Patients with FMF were divided to 3 groups as follows; Patients with typical compound heterozygous mutations of MEFV (E148Q/M694I) which indicated exon 10 mutation, were 3 cases (group 1). Patients with atypical mutations, except for mutations in exon 10, such as exon 1 (E84K, L110P), 2 (E148Q), 3 (P369S, R408Q), 5(S503C) and 9(I591M) were 8 cases (group 2) Patients with no mutations in MEFV gene were 9 cases (group 3). There were no significant differences of age at first visiting hospital (FV), onset age of fever attack (O), duration of fever attack (D) and frequency of fever attack (FF) between group 1, group2 and group 3 ( FV: $22.3 \pm$ $4.5,26.8 \pm 14.6$ years old (yo) and $29.5 \pm 8.7$ yo, $0: 11.6 \pm 2.4$ yo $18.5 \pm 13.8$ yo and $18.5 \pm 12.0 \mathrm{D}: 3.0 \pm 1.4 \mathrm{hrs}, 6.3 \pm 3.2 \mathrm{hrs}$, and $8.1 \pm 8.5 \mathrm{hrs}$, FF: $1.2 \pm 0.2 /$ month $(\mathrm{M}), 1.2 \pm 1.1 / \mathrm{M}$, and $1.1 \pm 0.2 / \mathrm{M})$ respectively. Laboratory examinations such as WBC, CRP and serum amyloid A (SAA) were not significantly different between 3 groups. All of those patients were effective for colchicine treatment except for 2 patients in group 1 because of severe diarrhea and alopecia. Finally, 2 patients in group 1 received canakinumab treatment. Mutations of $\mathrm{E} 148 \mathrm{Q}$ were found in 9 patients (45\%).

Conclusion: We have examined association between clinical features and mutations of MEFV in 20 Japanese patients, suggesting no positive findings in Japanese patients with FMF. Mutations of $\mathrm{E} 148 \mathrm{Q}$ in exon 2 were observed in $16-23 \%$ of normal Japanese patients ${ }^{1}$, indicating that $E 148 Q$ is the polymorphism or accelerating factor.

\section{REFERENCES}

[1] K. Migita et al, Familial Mediterranean fever : overview of pathogenesis, clinical features and management. Immunological Medicine 41:2, 55-61, 2018

Disclosure of Interests: None declared DOI: 10.1136/annrheumdis-2019-eular.1327

\begin{tabular}{|l|l|}
\hline AB0989 & PATIENTS' AND CAREGIVERS' ASSESSMENT OF A \\
& DEDICATED OUTPATIENT SERVICE FOR \\
& INTRAARTICULAR GLUCOCORTICOID INJECTIONS IN \\
& JUVENILE IDIOPATHIC ARTHRITIS
\end{tabular}

Hanan Jadoun ${ }^{1}$, Aurora Pucacco ${ }^{1}$, Angela Aquilani ${ }^{1}$, Andrea Uva ${ }^{2}$, Fabio Basta $^{1}$, Rebecca Nicolai ${ }^{1}$, Fabrizio De Benedetti ${ }^{1}$, Silvia Magni-Manzoni ${ }^{1}{ }^{1}$ Bambino Gesù Children's Hospital, Rheumatology, Rome, Italy; ${ }^{2}$ Sapienza University of Rome, Roma, Italy

Background: Patients with juvenile idiopathic arthritis (JIA) may require several hospital admissions over the disease course, due to flares or persistently active arthritis, with a negative impact on the patients' and family's daily life. To provide timely intervention and support the patients and families' quality of life, in 2018 an afternoon outpatient service for intraarticular glucocorticoid injections (IAGI) in JIA has been created at the study center.

Objectives: To evaluate the patients' and caregivers' assessment of the outpatient service for IAGI in JIA; to investigate demographic and clinical features of patients entering the service.

Methods: All consecutive JIA patients and their caregivers seen at the IAGI outpatient service from February 2018 to January 2019 completed a satisfactory questionnaire just after the IAGI procedure. The patient's par included: satisfaction on the overall service and on dedicated personnel (yes/no, why), procedure pain assessment (VAS 0-10, 0=none; 10 worst) whereas the caregiver's part: satisfaction on the overall service (yes/no, why), facilitation of family burden (yes/no, why). Demographic and clinical data of patients, including previous hospitalization for $|A G|$ under genera sedation or local anesthesia and geographical provenance, were registered during the questionnaire completion. Descriptive analysis was performed on data. Open answers were synthetized in items. 